\title{
A Dialog Management Methodology Based on Neural Networks and Its Application to Different Domains ${ }^{\star}$
}

\author{
D. Griol, L.F. Hurtado, E. Segarra, and E. Sanchis \\ Departament de Sistemes Informàtics i Computació (DSIC) \\ Universitat Politècnica de València (UPV) \\ Camí de Vera s/n, 46022 València, Spain \\ \{dgriol, lhurtado, esegarra, esanchis\}@dsic.upv.es
}

\begin{abstract}
In this paper, we present a statistical approach for dialog management within the framework of two different domains. The dialog model, that is automatically learned from a data corpus, is based on the use of a classification process to generate the next system answer. A neural network classifier is used for the selection process. This methodology has been applied in a spoken dialog system that provides railway information. The definition of an extended methodology that takes into account new system functionalities and its application for developing a dialog system for booking sports facilities is also described.
\end{abstract}

Keywords: Neural networks, Dialog systems, Dialog management, Statistical methodologies.

\section{Introduction}

The use of statistical techniques for the development of the different modules that compose a dialog system has been of growing interest during the last years [1]. These approaches are usually based on modeling the different processes in a probabilistic form and learning the necessary parameters from a dialog corpus. This type of methodologies has been traditionally applied within the fields of automatic speech recognition and natural language understanding [2], [3], [4]. The application of statistical methodologies to model the behavior of the dialog manager is providing interesting results in more recent years [5], [6], [7].

In this last field, we have developed an approach to manage the dialog using a statistical model learned from a labeled dialog corpus [8]. This work has been carried out within the framework of the DIHANA project [9]. The task that was considered for this project was the telephone access to a system that provides railway information in Spanish.

In this paper, we describe the adaptation of this methodology to manage tasks that involve the use of additional information not considered in our initial approach. The extended methodology has been applied for the development of a dialog manager for a new project called EDECAN [10]. One of the tasks defined within the framework of the

\footnotetext{
* Work partially supported by the Spanish MEC and FEDER under contract TIN2005-08660C04-02.
} 
EDECAN project is the development of a system for the booking of the sports facilities in our University.

Two different dialog corpora acquired for the specific tasks have been used for learning an initial dialog manager for the DIHANA and EDECAN tasks, whose evaluation is presented in this paper.

\section{Our Dialog Management Methodology for Slot-Filling Tasks}

In most dialog systems, the dialog manager takes its decisions based only on the information provided by the user in the previous turns and its own model. This is the case of most of slot-filling dialog systems. The methodology that we propose for the selection of the next system answer in this kind of tasks is as follows [8]:

Let $A_{i}$ be the output of the dialog system (the system answer) at time $i$, expressed in terms of dialog acts. Let $U_{i}$ be the semantic representation of the user turn (the result of the understanding process of the user input) at time $i$, expressed in terms of frames. We represent a dialog as a sequence of pairs (system-turn, user-turn):

$$
\left(A_{1}, U_{1}\right), \cdots,\left(A_{i}, U_{i}\right), \cdots,\left(A_{n}, U_{n}\right)
$$

where $A_{1}$ is the greeting turn of the system, and $U_{n}$ is the last user turn. We refer to a pair $\left(A_{i}, U_{i}\right)$ as $S_{i}$, the state of the dialog sequence at time $i$.

In this framework, we consider that, at time $i$, the objective of the dialog manager is to find the best system answer $A_{i}$. This selection is a local process for each time $i$ and takes into account the previous history of the dialog, that is to say, the sequence of states of the dialog preceding time $i$ :

$$
\hat{A}_{i}=\underset{A_{i} \in \mathcal{A}}{\operatorname{argmax}} P\left(A_{i} \mid S_{1}, \cdots, S_{i-1}\right)
$$

where set $\mathcal{A}$ contains all the possible system answers.

As the number of all possible sequences of states is very large, we define a data structure in order to establish a partition in the space of sequences of states (i.e., in the history of the dialog preceding time $i$ ). This data structure, that we call Dialog Register $(D R)$, contains the information provided by the user throughout the previous history of the dialog. All the information captured by the $D R_{i}$ at a given time $i$ is a summary of the information provided by the sequence $S_{1}, \cdots, S_{i-1}$. Using the $D R$, we obtain a great reduction in the number of different histories in the dialogs at the expense of a loss in the chronological information. We consider this to be a minor loss because the order in which the information is supplied by the user is not a relevant factor in determining the next system answer $A_{i}$. After applying the above considerations and establishing the equivalence relation in the histories of dialogs, the selection of the best $A_{i}$ is given by:

$$
\hat{A}_{i}=\underset{A_{i} \in \mathcal{A}}{\operatorname{argmax}} P\left(A_{i} \mid D R_{i-1}, S_{i-1}\right)
$$

The selection of the system answer is carried out through a classification process, for which a multilayer perceptron (MLP) is used. The input layer receives the codification of the pair $\left(D R_{i-1}, S_{i-1}\right)$. The output generated by the MLP can be seen as the probability of selecting each one of the different system answers defined for a specific task. 


\subsection{Application of This Methodology to the DIHANA Project}

The DIHANA project undertook the design and development of a dialog system for the access to an information system using spontaneous speech. The domain of the project was the query to an information system about railway timetables, prices and services in Spanish by telephone. The main goal of the project was the development of a robust, distributed and modular dialog system for access to information systems.

The representation of user and system turns in the DIHANA project is done in terms of dialog acts. In the case of user turns, the dialog acts correspond to the classical frame representation of the meaning of the utterance. In this task, we defined eight concepts (Hour, Price, Train-Type, Trip-Time, Services, Affirmation, Negation, and Not-Understood) and ten attributes (Origin, Destination, Departure-Date, ArrivalDate, Departure-Hour, Arrival-Hour, Class, Train-Type, Order-Number and, Services). An example of the semantic interpretation of an input sentence is shown below:

\section{User Turn:}

Yes, I would like to know the timetables leaving from Barcelona.

\section{Semantic interpretation:}

(Affirmation)

(Hour)

Origin: Barcelona

Three levels of labeling were defined for the system dialog acts. The first level describes the general acts of any dialog, independently of the task. The second level represents the concepts involved in the turn and is specific to the task. The third level represents the values of the attributes given in the turn. The following labels were defined for the first level: Opening, Closing, Undefined, Not-Understood, Waiting, New-Query, Acceptance, Rejection, Question, Confirmation, and Answer. The labels defined for the second and third level were the following: Departure-Hour, Arrival-Hour, Price, TrainType, Origin, Destination, Date, Order-Number, Number-Trains, Services, Class, TripType, Trip-Time and, Nil. An example of the labeling of a system turn is shown below:

\section{System Turn:}

Do you want to know timetables?

\section{Semantic interpretation:}

(Confirmation:Departure-Hour:Nil)

For the DIHANA task, the $D R$ is a sequence of 15 fields, where each concept or attribute has a field associated to it. The sequence of fields for concepts is Hour, Price, Train-Type, Trip-Time, and Services. The sequence of fields for attributes is Origin, Destination, Departure-Date, Arrival-Date, Departure-Hour, Arrival-Hour, Class, TrainType, Order-Number and, Services.

Following the methodology described in this section, Figure 1 shows the operation of the dialog manager developed for the DIHANA project. The frames generated by the understanding module after each user turn and the last system answer are used to generate the pair $\left(D R_{i-1}, S_{i-1}\right)$. The codification of this pair is the input of a MLP that 


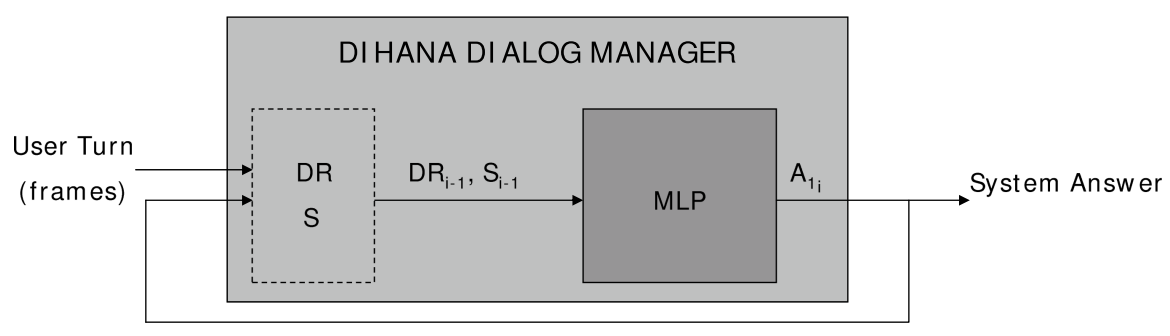

Fig. 1. Scheme of the dialog manager designed for the DIHANA project

provides the probabilities of selecting each one of the system answers defined for the DIHANA task, given the current situation of the dialog (represented by this pair).

\section{Dialog Management in Tasks Involving an Application Manager}

In other dialog systems, the dialog manager generates the following system answer taking into account not only the information provided by the user, but also the information generated by the module that controls the application (that we call Application Manager, $A M)$. The $A M$ usually performs two main functions. On the one hand, this module performs the queries to the database. On the other hand, it has to verify if the user query follows the regulations defined for the task. Then, the result of queries to the $A M$ has to be considered in order to generate the following system answer.

We have decided that two phases are required for the selection of the next system turn in this type of tasks. In the first phase, the information contained in the $D R$ and the last state $S_{i-1}$ are considered to select the best request to be made to the $A M\left(\hat{A}_{1_{i}}\right)$ :

$$
\hat{A}_{1_{i}}=\underset{A_{1_{i}} \in \mathcal{A}_{1}}{\operatorname{argmax}} P\left(A_{i} \mid D R_{i-1}, S_{i-1}\right)
$$

where $\mathcal{A}_{1}$ is the set of possible requests to the $A M$.

In the second phase, the final system answer $\left(\hat{A}_{2_{i}}\right)$ is generated taking into account $\hat{A}_{1_{i}}$ and the information provided by the $A M\left(A M_{i}\right)$ :

$$
\hat{A}_{2_{i}}=\underset{A_{2_{i}} \in \mathcal{A}_{2}}{\operatorname{argmax}} P\left(A_{i} \mid A M_{i}, A_{1_{i}}\right)
$$

where $\mathcal{A}_{2}$ is the set of possible system answers.

We propose the use of two MLPs to carry out the selection of the next system answer, each one to deal with the specific information defined for each phase.

\subsection{Application of the Extended Methodology to the EDECAN Project}

The main objective of the EDECAN project is to increase the robustness of a spontaneous speech dialog system through the development of technologies for the adaptation and personalization of the system to the different acoustic and application contexts in 
which it can be used. The task defined for the EDECAN project is the multilingual query to an information system for the booking of sport activities in our University. Users can ask for the availability, the booking or cancellation of facilities and the information about their current bookings.

The definition of the semantics of the EDECAN task has been carried out considering the different functionalities required for the booking system and the information that is required to complete them. In the case of user turns, we defined seven concepts (Availability, Booking, Booked, Cancellation, Affirmation, Negation, and Not-Understood) and six attributes (Sport, Hour, Date, Court-Type, Court-Number, and Order-Number). An example of the semantic interpretation of an input sentence is shown below:

\section{User Turn:}

I want to book a tennis court for tomorrow.

\section{Semantic Representation:}

(Booking)

Sport: tennis

Date: tomorrow

The labeling of the system turns is similar to the labeling defined for the user turns. A total of 25 task-dependent concepts has been defined: Task-independent concepts (Affirmation, Negation, Not-Understood, New-Query, Opening, and Closing); concepts used to inform the user about the result of a specific query (Availability, Booking, Booked, and Cancellation), concepts defined to require the user the attributes that are necessary for a specific query (Sport, Date, Hour, and Court-Type), concepts used for the confirmation of concepts (Confirmation-Availability, Confirmation-Booking, Confirmation-Booked, Confirmation-Cancellation) and attributes (Confirmation-Sport, Confirmation-Date, Confirmation-Hour, Confirmation-CourtType), and concepts relative to the AM (Rule-Info, Booking-Choice, and Cancellation-Choice).

A total of six attributes has been defined (Sport, Court-Type, Court-Number, Date, Hour, and Availability-Number). An example of the labeling of a system turn is shown below:

\section{System Turn:}

Do you want to book a paddle court from 20:00 to 20:30?

\section{Labeling:}

(Confirmation-Booking)

Sport: paddle

Hour: 20:00-20:30

The $D R$ defined for the EDECAN stores a total of ten characteristics, corresponding to the four concepts and six task-dependent attributes defined for the labelling of the user turns. The answer generated by the AM has been coded taking into account five possible cases: the AM has not taken part for the generation of the system answer (case 1), the AM provides that there is not any available facility (case 2), there is only one facility that is available (case 3 ) or more than one facility are available (case 4), the user query does not fulfill the regulations specified in our University (case 5). 


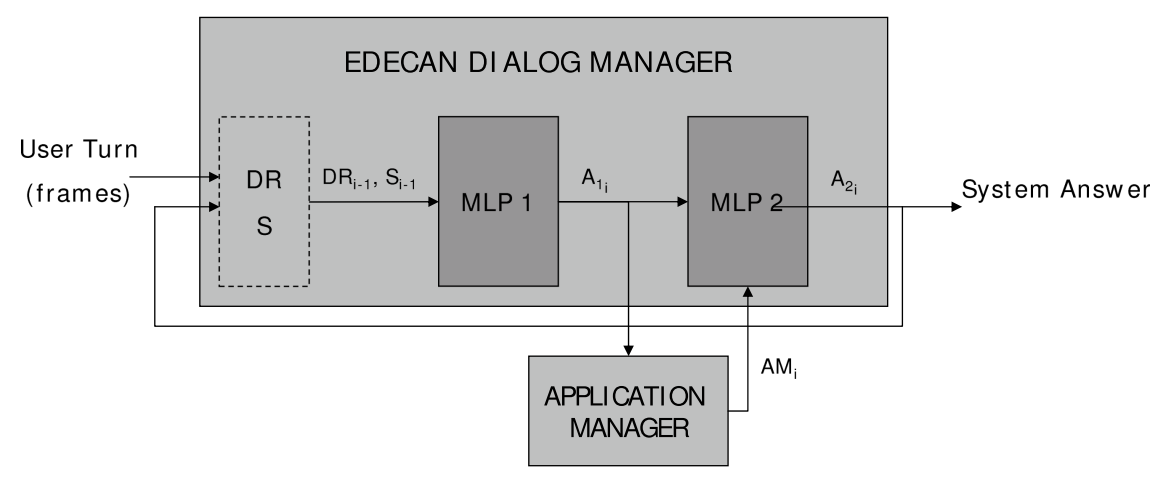

Fig. 2. Scheme of the dialog manager designed for the EDECAN project

Figure 2 shows the scheme proposed for the development of the dialog manager for the EDECAN project, detailing the two phases described for the generation of the final system answer.

\section{Evaluation}

The evaluation of the dialog managers developed for the DIHANA and EDECAN projects was carried out using a cross validation process. A corpus of 900 dialogs acquired by means of the Wizard of $\mathrm{Oz}$ technique was used to develop and evaluate the DIHANA dialog manager. For the construction and evaluation of the EDECAN dialog manager, we used a corpus of 150 dialogs recorded at the telephone sport service of our University with real users.

Our experiment consisted of five trials. Each trial used a different subset taken from the five subsets as the test set, and the remaining $80 \%$ of the corpus was used as the training set. Software developed in our labs was used to model and train the MLPs. A validation subset (20\%) was extracted from each training set. MLPs were trained using the backpropagation with momentum algorithm [11]. The best topology was two hidden layers with 100 and 10 neurons

The evaluation was carried out turn by turn. Three measures were defined to carry out this evaluation:

- Percentage of answers that are equal to the reference answer in the corpus (\% exact).

- Percentage of answers that are coherent with the current state of the dialog (\%correct).

- Percentage of answers that are not coherent with the current state of the dialog (\%incoherent), but do not produce the failure of the dialog.

- Percentage of answers that are not coherent with the current state of the dialog (\%error), and would cause the failure of the dialog.

These two last measures have been obtained after a manual revision of the answers provided by the dialog manager. 
Table 1 shows the results of the evaluation of the dialog manager developed for the DIHANA project. These results show the satisfactory operation of the developed dialog manager. The codification developed to represent the state of the dialog and the good operation of the MLP classifier make it possible for the answer generated by the manager to be exactly the reference answer (\%exact) in $76.62 \%$ of the cases.

Moreover, the number of answers generated by the MLP that can cause the failure of the system is only $0.42 \%$ percentage. An answer that is coherent with the current state of the dialog is generated in $99.33 \%$ of cases. These last two results also demonstrate the correct operation of the classification methodology.

Table 1. Results of the evaluation of the dialog manager developed for the DIHANA project

\begin{tabular}{|c|c|c|c|c|}
\hline & \%exact & \%correct & \%incoherent & \%error \\
\hline System answer & $76.62 \%$ & $99.33 \%$ & $0.25 \%$ & $0.42 \%$ \\
\hline
\end{tabular}

Table 2 shows the results of the evaluation of the EDECAN dialog manager. The results obtained after the experimentation show that the dialog manager correctly adapts to the requirements of the new task, providing $86.75 \%$ of answers that are coherent with the current state of the dialog, and a $72.91 \%$ percentage of answers are equal to the reference answer in the corpus.

The percentage of answers provided by the manager that can cause the failure of the dialog is very high $(4.56 \%)$.

Besides, a $8.69 \%$ percentage of the answers do not produce the failure of the dialog but are not coherent with its current state (e.g. system answers that require information already provided by the user). We want to reduce this percentage by means of incorporating new dialogs to our initial corpus.

Table 2. Results of the evaluation of the dialog manager developed for the EDECAN project

\begin{tabular}{|c|c|c|c|c|}
\hline & \%exact & \%correct & \%incoherent & \%error \\
\hline System answer & $72.91 \%$ & $86.75 \%$ & $8.69 \%$ & $4.56 \%$ \\
\hline
\end{tabular}

\section{Conclusions}

In this paper, we have presented an approach for the development of a statistical dialog manager. We have developed a detailed representation of the user and system dialog acts. This representation allows the system to automatically generate a specialized answer that takes into account the current situation of the dialog. From this representation, a classification methodology based on MLPs is used in order to generate the system answers.

By means of this methodology and its extended adaptation, we have developed two dialog managers. Some experiments have been performed to evaluate this methodology within the framework of two different tasks. The results of the evaluation show the satisfactory operation of the developed approach, with the possibility of improving the initial dialog model by means of the incorporation of new dialogs. 
At the moment, our objective is to carry out the evaluation of the different modules that make up the EDECAN dialog system with real users. This evaluation is going to be made in a supervised way, using the statistical dialog manager presented in this work. The acquired dialogs will be used to evaluate and improve the initial dialog model.

\section{References}

1. Young, S.: The Statistical Approach to the Design of Spoken Dialogue Systems. Technical report, CUED/F-INFENG/TR.433, Cambridge University Engineering Department (2002)

2. Segarra, E., Sanchis, E., Galiano, M., García, F., Hurtado, L.: Extracting Semantic Information Through Automatic Learning Techniques. International Journal on Pattern Recognition and Artificial Intelligence 16(3), 301-307 (2002)

3. He, Y., Young, S.: A data-driven spoken language understanding system. In: Proc. of ASRU 2003, pp. 583-588 (2003)

4. Esteve, Y., Raymond, C., Bechet, F., Mori, R.D.: Conceptual Decoding for Spoken Dialog systems. In: Proc. of EuroSpeech 2003, pp. 617-620 (2003)

5. Williams, J., Young, S.: Partially Observable Markov Decision Processes for Spoken Dialog Systems. Computer Speech and Language 21(2), 393-422 (2007)

6. Lemon, O., Georgila, K., Henderson, J.: Evaluating Effectiveness and Portability of Reinforcement Learned Dialogue Strategies with real users: the TALK Town-Info. Evaluation. In: Proc. of SLT 2006, Aruba (2006)

7. Torres, F., Sanchis, E., Segarra, E.: Development of a stochastic dialog manager driven by semantics. In: Proc. EuroSpeech 2003 (1), 605-608 (2003)

8. Hurtado, L., Griol, D., Segarra, E., Sanchis, E.: A Stochastic Approach for Dialog Management based on Neural Networks. In: Proc. of InterSpeech 2006, Pittsburgh (2006)

9. Benedí, J., Lleida, E., Varona, A., Castro, M., Galiano, I., Justo, R., López, I., Miguel, A.: Design and acquisition of a telephone spontaneous speech dialogue corpus in Spanish: Dihana. In: Proc. of LREC 2006, Genove (2006)

10. Lleida, E., Segarra, E., Torres, M., Macías-Guarasa, J.: EDECÁN: sistEmade Diálogo multidominio con adaptación al contExto aCústico y de AplicacióN. In: Proc. IV Jornadas en Tecnologia del Habla, Zaragoza, pp. 291-296 (2006)

11. Rumelhart, D.E., Hinton, G.E., Williams, R.J.: Learning internal representations by error propagation. In: PDP: Computational models of cognition and perception, vol. I, pp. 319362. MIT Press, Cambridge (1986) 